\title{
LE CONTEXTE DE LA PANDÉMIE MONDIALE COMME POSSIBLE SOURCE D'INNOVATION : APPRENTISSAGE EXPANSIF ET RÉSOLUTION DE CONTRADICTIONS
}

\author{
Aude Gagnon-Tremblay \\ Étudiante à la maitrise en psychopédagogie \\ Université Laval
}

Jessy Turcotte

Étudiante à la maitrise en didactique

Université Laval

\author{
aude.gagnon-tremblay.1@ulaval.ca
}

\author{
jessy.turcotte.2@ulaval.ca
}

\begin{abstract}
Résumé
La fermeture des écoles causée par la pandémie de la COVID-19 a suscité beaucoup d'inquiétude dans les milieux éducatifs. En effet, les élèves n'ont plus eu accès à l'enseignement traditionnel dans les établissements scolaires. Les enseignants ont dû s'adapter rapidement aux nouvelles mesures d'urgence et enseigner à distance, en mode virtuel. En plus de devoir s'approprier une variété d'outils numériques, ils ont été soumis à une pression quant à la qualité de leur enseignement et à l'encadrement des élèves. Le climat inhabituel et incertain causé par la pandémie a donc amené plusieurs acteurs du milieu scolaire à réfléchir à leur pratique afin d'améliorer l'expérience éducative des élèves lors d'un futur retour en classe. Ce changement obligatoire de stratégie pédagogique représente un défi susceptible de créer des tensions chez les enseignants qui ont décidé d'utiliser de nouvelles approches pédagogiques davantage centrées sur l'élève. Cet article, ancré dans la troisième génération de la théorie de l'activité, se base sur une recension systématique des écrits et analyse la façon dont les défis engendrés par l'enseignement virtuel ont pu favoriser l'apprentissage expansif des enseignants. Cette revue de la documentation suggère de possibles contradictions représentant les quatre niveaux établis par Engeström (2001) et propose des étapes susceptibles de mener à une transformation durable de l'activité. En conclusion, il avance que la résolution des contradictions vécues par les enseignants favorisera à la fois la transformation de leurs pratiques pédagogiques ainsi que leur adaptation à la nouvelle réalité virtuelle.
\end{abstract}

Mots-clés : COVID-19, apprentissage expansif, contradictions, pratiques enseignantes, théorie de l'activité

\begin{abstract}
The closure of schools caused by the COVID-19 pandemic has caused a great deal of concern in educational circles. Indeed, students no longer had access to traditional education in schools. Teachers had to adapt quickly to the new emergency measures and teach remotely, in virtual mode. In addition to having to learn a variety of digital tools, they were under pressure in terms of the quality of their teaching and the supervision of students. The unusual and uncertain climate caused by the pandemic has therefore led several actors in the school community to reflect on their practice to improve the educational experience of students for the future return
\end{abstract}


Revue internationale du CRIRES: innover dans la tradition de Vygotsky (2021) ISSN 2291-6717, vol 5, no 2, 63-73

Le contexte de la pandémie mondiale comme source possible d'innovation: apprentissage expansif et résolution de contradictions

to class. This mandatory change in pedagogical strategy represents a challenge likely to create tensions among teachers who have decided to use new, more student-centered pedagogical approaches. This article, anchored in the third generation of activity theory, is based on a systematic review of the literature, and analyzes how the challenges generated by virtual teaching have been able to promote the expansive learning of teachers. This review of the literature suggests possible contradictions representing the four levels established by Engeström (2001) and proposes steps likely to lead to a sustainable transformation of the activity. In conclusion, it argues that the resolution of the contradictions experienced by teachers will promote both the transformation of their teaching practices as well as their adaptation to the new virtual reality.

Keywords: COVID-19, expansive learning, activity theory, teaching practices, contradictions.

\section{Note d'auteur}

Toute correspondance concernant cet article doit être adressée à Aude GagnonTremblay (aude.gagnon-tremblay.1@ulaval.ca) ou à Jessy Turcotte (jessy.turcotte.2@ulaval.ca). 
Revue internationale du CRIRES: innover dans la tradition de Vygotsky (2021) ISSN 2291-6717, vol 5, no 2, 63-73 Le contexte de la pandémie mondiale comme source possible d'innovation: apprentissage expansif et résolution de contradictions

\section{Introduction}

Cet article a pour objet de décrire l'apprentissage expansif possible des enseignants dans le contexte de la pandémie mondiale provoquée par la maladie à coronavirus (COVID-19). Cette maladie unique et inquiétante a amené le Canada, puis le Québec, à déclarer l'état d'urgence sanitaire le 14 mars $2020^{1}$ afin de limiter la propagation du virus (Gouvernement du Québec, 2020). Cette mesure exceptionnelle a entraîné un arrêt quasi total de l'économie, des déplacements à l'étranger et de la vie «normale ». En plus d'interdire les rassemblements extérieurs et intérieurs, autant privés que publics, et les visites dans les hôpitaux et les CHSLD, l'état d'urgence a aussi forcé la fermeture des lieux non essentiels comme les commerces, les restaurants et même les établissements scolaires. L'UNICEF déplore d'ailleurs la fermeture des écoles. Selon la directrice des politiques et des programmes internationaux d'UNICEF Canada, «dans les contextes d'urgence, les écoles sont souvent "les premiers services à être interrompus... Et parallèlement, elles sont très souvent parmi les derniers services à reprendre" » (Gouvernement du Canada, 2021). Plusieurs craignent aussi que cette fermeture donne lieu «à des dommages irréversibles et à une "génération perdue" de jeunes » (Gouvernement du Canada, 2021). D'ailleurs, des données montrent que depuis le début de la pandémie, plus de 1,5 milliard d'élèves ont vu leur apprentissage perturbé, ce qui représente près de $90 \%$ des élèves mondiaux (Gouvernement du Canada, 2021).

\section{Problématique}

En plus de s'inquiéter pour leur santé et leur sécurité et celles de leurs élèves, les enseignants ont dû se mobiliser rapidement lors de la fermeture des écoles pour trouver des façons alternatives d'interagir avec les jeunes et de les scolariser (Mullen et al., 2021). En effet, les mesures mises en œuvre par les gouvernements pour contrer la propagation de la COVID19 ont forcé une transition «spontanée » et non prévue de l'enseignement en présentiel à l'enseignement virtuel ${ }^{2}$, laissant peu de temps aux enseignants pour se former adéquatement (Gopalan et al., 2021 ; Seke, 2020; Westwick \& Morreale, 2020). Pouvant se dérouler de manière synchrone (en temps réel), asynchrone (à l'aide d'enregistrements) ou hybride, l'enseignement virtuel utilise les technologies informatiques pour favoriser les apprentissages des élèves (Besser et al., 2020; Syauqi et al., 2020). Afin d'être efficace, ce type d'enseignement doit être planifié systématiquement et l'enseignant doit avoir des connaissances préalables en la matière (Durak \& Çankaya, 2020). Autrement dit, le facteur temps est important dans l'implantation de l'enseignement virtuel (Özüdogru, 2021). Toutefois, étant donné les mesures gouvernementales, les enseignants n'ont eu d'autres choix que de s'approprier rapidement et de manière autonome différentes technologies, parfois inconnues, afin de continuer à enseigner (Nations Unies, 2020). De surcroît, certains ont aussi dû composer avec un manque de ressources humaines et matérielles (UNESCO, 2020).

Devant l'urgence de s'adapter à la nouvelle réalité de l'enseignement à distance, plusieurs enseignants ont ressenti de la confusion, du stress et un sentiment d'incompétence (Kim \& Asbury, 2020). En effet, l'UNESCO (2020) rapporte que les enseignants ont ressenti une pression pour maintenir la qualité de leur enseignement et ont éprouvé de la difficulté à encadrer, à supporter et à évaluer les apprentissages des élèves tout en gérant les problèmes techniques qui surviennent lorsqu'ils enseignent. La forte demande imposée aux enseignants (Department for Education, 2020) et la vitesse à laquelle ils ont dû changer leurs pratiques contribueraient au climat d'incertitude provoqué par la pandémie (Kim \& Asbury, 2020).

\footnotetext{
${ }^{1}$ À cette date, il y avait 21 cas confirmés de personnes atteintes de la COVID-19 au Québec (gouvernement du Québec, 2020).

${ }^{2}$ L'enseignement virtuel se nomme aussi enseignement à distance ou en ligne.
} 


\section{Concepts Clés et Question de Recherche}

Bien que le contexte de la pandémie s'avère difficile et éprouvant pour les enseignants (Kim \& Asbury, 2020), il peut aussi leur offrir l'occasion de dépasser certains conflits dans le but d'innover leurs pratiques enseignantes. Les conflits vécus peuvent entrainer les enseignants utilisant une approche plus traditionnelle, moins centrée sur l'élève, à réfléchir ou à changer de posture afin de stimuler l'apprentissage des apprenants (Berger \& Paul, 2021). La théorie de l'apprentissage expansif est un concept issu de la troisième génération de la théorie de l'activité développée par Engeström (1987) qui permet de comprendre la façon dont les contradictions peuvent constituer une source puissante d'apprentissage, de transformation et d'innovation. Par la résolution de conflits et la création de nouveaux savoirs, l'apprentissage expansif transforme de manière durable et profonde la pratique (Engeström \& Pyorala, 2021 ; Engeström \& Sannino, 2010).

Cet article se base sur une recension systématique des écrits traitant de l'apprentissage expansif et répond à la question suivante : le contexte de la pandémie de la COVID-19 peut-il favoriser l'apprentissage expansif des enseignants par l'entremise de la résolution des contradictions?

\section{Méthodologie}

Trouver une réponse à cette question a demandé deux recherches systématiques de mots-clés. Ces recherches ont été réalisées un an après la déclaration de l'état d'urgence sanitaire par les gouvernements. Durant cette période, de nombreux articles traitant des impacts de la fermeture des écoles sur les enseignants ont été publiés. La première recherche a été effectuée sur trois bases de données importantes dans le domaine de l'éducation (Academic Search Premier, ERIC et Education Source) et recherchait les mots-clés Expansive learning et activity theory ainsi que sur une base de données francophone qui regroupe des articles de revues scientifiques publiés au Québec et en France couvrant les domaines des sciences humaines et sociales (Érudit) et recherchait les mots-clés Apprentissage expansif et Théorie de l'activité. Afin d'être sélectionnés, les articles devaient mentionner les mots-clés dans leur titre, leur résumé ou leurs mots-clés et avoir été évalués par les pairs (peer-reviewed articles) (voir Tableau 1).

\section{Tableau 1}

Recension systématique des écrits

\begin{tabular}{|l|l|l|c|}
\hline \multicolumn{1}{|c|}{ Bases de } & \multicolumn{1}{|c|}{ Mots-clés } & \multicolumn{1}{|c|}{ Filtres } & N \\
\hline données & & & \\
\hline Academic & $\begin{array}{l}\text { Expansive } \\
\text { learch } \\
\text { learning ET } \\
\text { ERIC },\end{array}$ & $\begin{array}{l}\text { Peer reviewed ; } \\
\text { Mots-clés dans le résumé ; } \\
1980 \text { à 2021. }\end{array}$ & 179 \\
\hline
\end{tabular}




\begin{tabular}{|l|l|l|c|}
\hline $\begin{array}{l}\text { Education } \\
\text { Source }\end{array}$ & & \\
\hline Érudit & $\begin{array}{l}\text { Apprentissage } \\
\text { expansif ET } \\
\text { théorie de } \\
\text { l'activité }\end{array}$ & $\begin{array}{l}\text { Articles savants; } \\
\text { Articles ; } \\
\text { Mots-clés dans le titre, résumé ou } \\
\text { mots-clés; } \\
\text { Français; } \\
1980-2021 .\end{array}$ & 131 \\
& & & \\
\hline
\end{tabular}

La seconde recension a été effectuée dans les articles recherchés précédemment à l'aide de deux nouvelles catégories de mots-clés (voir Tableau 2). La première catégorie regroupe les articles qui abordent les fondements théoriques de l'apprentissage expansif et de la théorie de l'activité alors que la seconde regroupe les articles qui traitent du milieu de l'éducation ou des pratiques enseignantes. Les articles ne mentionnant pas ces mots-clés ont été rejetés.

\section{Tableau 2}

Tri et catégorisation des articles

\begin{tabular}{|c|c|c|}
\hline Bases de données & $\begin{array}{c}\text { Mots-clés ajoutés aux filtres déterminés } \\
\text { précédemment }\end{array}$ & $\mathbf{n}$ \\
\hline \multirow{2}{*}{$\begin{array}{l}\text { Academic Search } \\
\text { Premier, ERIC, } \\
\text { Education Source }\end{array}$} & 1. Theoretical framework & 11 \\
\hline & $\begin{array}{l}\text { 2. Teaching strategies OU teaching methods OU } \\
\text { teaching approaches OU classroom techniques }\end{array}$ & 25 \\
\hline \multirow[t]{2}{*}{ Érudit } & 1. Fondements théoriques & 13 \\
\hline & $\begin{array}{l}\text { 2. Pratiques didactiques OU éducation OU } \\
\text { enseignant }\end{array}$ & 58 \\
\hline
\end{tabular}

Au terme de cette deuxième recherche systématique, les articles satisfaisant les critères ont été lus attentivement. En tout, 30 articles pertinents ont été conservés pour l'analyse des possibilités d'innovation des pratiques enseignantes post-COVID-19 dans les pays occidentaux, particulièrement le Canada, la France et le Royaume-Uni, sous l'angle de l'apprentissage expansif.

\section{Analyse des écrits}

\section{L'apprentissage expansif et la théorie de l'activité}


Revue internationale du CRIRES: innover dans la tradition de Vygotsky (2021) ISSN 2291-6717, vol 5, no 2, 63-73 Le contexte de la pandémie mondiale comme source possible d'innovation: apprentissage expansif et résolution de contradictions

L'enseignement à distance est une approche pédagogique non traditionnelle qui est susceptible d'entrainer l'apparition de difficulté chez les enseignants peu familiers avec cette méthode (Kim \& Asbury, 2020). Justement, l'apprentissage expansif représente une méthode d'analyse et de promotion de l'apprentissage pour le changement dans des contextes non traditionnels, hybrides et multiorganisationnels (Engeström \& Pyorala, 2012 ; Engeström \& Sannino, 2010). L'apprentissage expansif constitue en un processus de construction partagé d'un nouvel objet en réponse à une contradiction dans une activité collective (Engeström \& Sannino, 2010). Cet apprentissage profond transformerait durablement l'activité d'un collectif par la création de nouveaux savoirs ou de nouvelles pratiques pour une activité émergente (Goodnough \& Murphy, 2017; Viau-Guay \& Hamel, 2017). Ce concept se situe dans la troisième génération de la théorie socioculturelle de l'activité (CHAT) (Engeström, 1999). Cette génération de la CHAT se fonde sur les deux générations précédentes, soit celle de Vygotsky (1985) et de Leontiev (1978).

La première génération de la théorie de l'activité est centrée autour des travaux de Vygotsky, un psychologue russe qui a développé le concept de médiation. Selon ce concept, le comportement d'un sujet n'est pas une réponse directe à un stimulus, mais est plutôt médié par différents outils symboliques (Brassac, 2003). En d'autres mots, le comportement humain est influencé par la culture du sujet, d'où l'importance de considérer le contexte dans lequel s'insère l'activité afin de comprendre comment se construisent les connaissances.

La deuxième génération de la théorie de l'activité est attribuée à Leont'ev, un auteur qui accorde davantage de place à la dimension collective de l'activité et qui distingue l'action individuelle de l'action collective (Engeström, 1999). Pour Leont'ev, la médiation se caractérise à la fois par les règles établies et par la division du travail dans une communauté d'individus qui partagent le même motif (Barma, 2008). Ce motif représente le but concret de l'activité. Il doit donc être significatif pour l'individu ou pour le groupe et viser la transformation de l'environnement.

La troisième génération considère l'activité comme un processus essentiellement collectif, mais lui donne un sens plus large que les deux générations précédentes. En effet, l'activité s'oriente maintenant vers un objet dont le rapport participe au développement d'une activité socialement réglée et médiatisée par des outils (Barma, 2010 ; Engeström, 2001). Elle s'attarde ainsi davantage à la transformation d'une collectivité qu'à la transformation d'un individu puisqu'elle reflète divers points de vue, traditions et intérêts. Aussi, afin d'accomplir leur motif, les individus doivent tenir compte de la division du travail au sein de leur communauté (distribution des tâches), des règles et normes ainsi que des outils (autant matériels que psychologiques) mis à leur disposition (Deslandes et al., 2020 ; Engeström, 1987). Finalement, cette troisième génération considère que pour bien comprendre et transformer les collectivités, il faut analyser les interactions entre plusieurs systèmes d'activité partageant un même objet (objet 3) et leur historicité (Barma, 2010 ; Engeström, 2001 ; Martinez-Alvarez, 2019). D'ailleurs, lors de la transformation, des contradictions peuvent émerger dans et entre les systèmes d'activité, et constituer une source puissante de changement (Engeström, 2001). 
Revue internationale du CRIRES: innover dans la tradition de Vygotsky (2021) ISSN 2291-6717, vol 5, no 2, 63-73

Le contexte de la pandémie mondiale comme source possible d'innovation: apprentissage expansif et résolution de contradictions

\section{Figure 1}

Interactions entre deux systèmes d'activités partageant un même objet (Engeström, 2001)

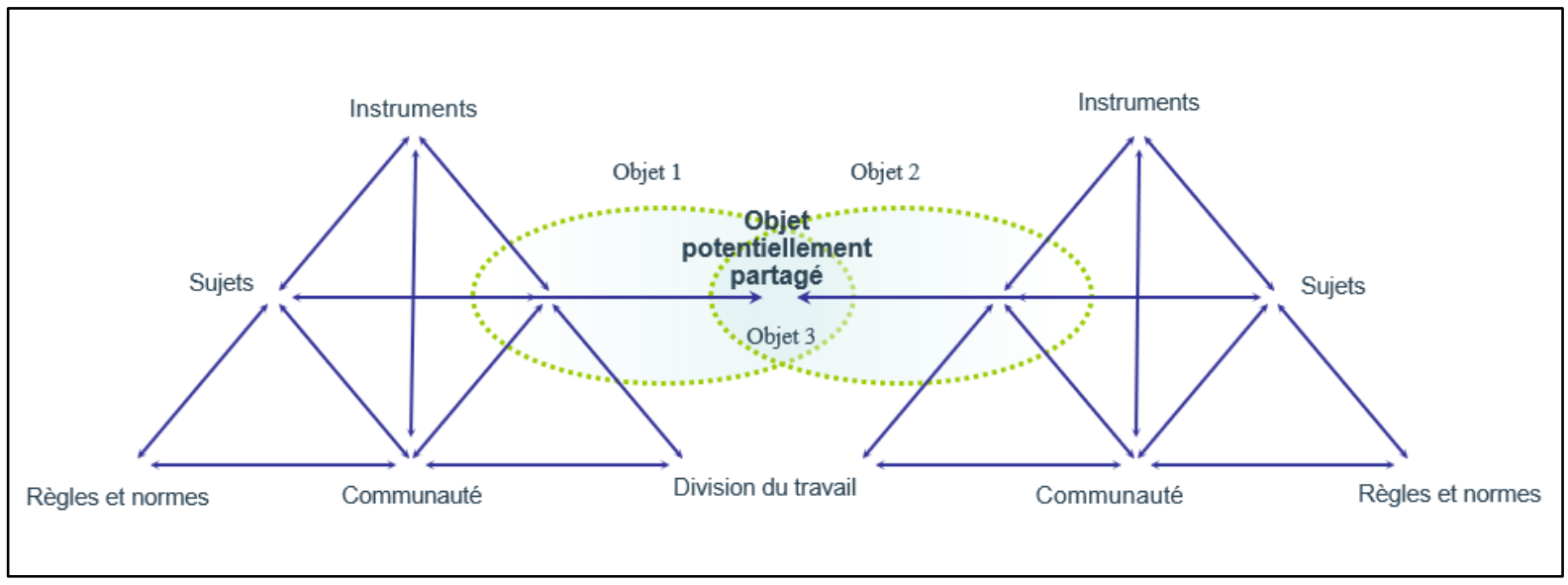

\section{Les contradictions et le cycle d'expansion}

Les contradictions entre les différents systèmes jouent un rôle central dans la troisième génération de la CHAT puisque leurs résolutions permettent les transformations expansives, comme le changement ou l'innovation (Barma, 2010; Engeström, 2001; Engeström \& Sannino, 2010). Plus précisément, les tensions cumulées au gré du temps deviennent des contradictions structurelles à l'intérieur et entre les systèmes d'activité. Leur aggravation permettrait aux individus de questionner et de s'éloigner des normes établies (Engeström \& Sannino, 2010 ; Sannino et al., 2016). L'innovation se produirait lorsque l'objet et le motif de l'activité sont conceptualisés pour adopter une étendue plus grande de possibilités que le mode d'activité précédent (Engeström, 2001; Goodnough \& Murphy, 2017; Martinez-Alvarez, 2019). Cette innovation est représentée par l'objet potentiellement partagé illustré dans la Figure 1.

\section{La résolution des contradictions vécues par les enseignants durant la pandémie}

Dans cette section, huit articles ont été utilisés pour analyser les contradictions ou les transformations expansives possibles qui peuvent émerger des difficultés éprouvées par les enseignants lors du passage obligatoire à l'enseignement à distance. L'interprétation des articles permettra de déterminer si le contexte de la pandémie est en mesure de contribuer à l'apprentissage expansif des enseignants.

Rappelons que la fermeture des établissements scolaires a amené plusieurs enseignants à réfléchir sur leur pratique et à trouver des alternatives efficaces afin de maintenir la qualité de leur enseignement (Koris \& Pal, 2021). Cette réflexion est susceptible d'avoir contribué à l'émergence de plusieurs contradictions par rapport à l'obligation d'enseigner à distance. Engeström (2001) relève quatre niveaux de contradictions possibles dans un système ou à travers les systèmes d'activité. Le premier niveau représente l'état de besoin. Avec le passage au virtuel, certains enseignants éprouvent de la difficulté à s'approprier les technologies, à interagir avec les élèves et à les engager dans leurs apprentissages (Kim \& Asbury, 2020 ; Mullen et al., 2021). Dans ce cas, le nouvel objet de l'activité peut être de trouver une méthode pour remédier à ces difficultés.

Le deuxième niveau concerne la double contrainte créée par la production d'un nouvel objet. La résolution de cette contradiction demande l'analyse globale de la situation pour 
Revue internationale du CRIRES: innover dans la tradition de Vygotsky (2021) ISSN 2291-6717, vol 5, no 2, 63-73 Le contexte de la pandémie mondiale comme source possible d'innovation: apprentissage expansif et résolution de contradictions

remettre en question le sens de l'activité (Barma, 2010 ; Deslandes et al., 2020). Ensuite, une solution peut être modélisée. À ce niveau, un enseignant pourrait développer une nouvelle forme de pratique qui engagerait davantage ses élèves dans les cours en ligne. Il pourrait aussi décider de s'éloigner de l'enseignement virtuel synchrone, qui est similaire à l'enseignement magistral traditionnel, et choisir une méthode plus collaborative, telle que l'apprentissage par problème ou par projet. Cette nouvelle orientation pédagogique est susceptible d'entrainer l'émergence d'autres contradictions entre la nouvelle pratique et la pratique dominante du milieu (Barma, 2010), ce qui représente le troisième niveau de contradiction. Par exemple, alors qu'avant la pandémie, une école accordait beaucoup d'importance à la performance scolaire, il est possible qu'en temps de pandémie, certains enseignants décident de prioriser davantage les apprentissages des élèves. Ainsi, si la pratique émergente n'a pas comme objectif l'évaluation des élèves, elle peut entrer en confrontation avec la culture du milieu et faire émerger des tensions chez l'enseignant qui désire changer ses pratiques.

S'il y a dépassement de cette troisième contradiction, l'implantation de la nouvelle pratique enrichie est facilitée. Cependant, de nouvelles tensions peuvent apparaître entre les différents systèmes d'activité. Ce dernier niveau de contradiction, le quatrième, se manifesterait entre l'enseignant, les parents et d'autres membres de l'école, par exemple. Même si ces acteurs possèdent globalement les mêmes visées, soit la réussite éducative des élèves, la vision des parents concernant les pratiques enseignantes adoptées en temps de pandémie peut être différente de celle de l'enseignant, créant ainsi un frein à l'innovation ou la transformation. Afin de surmonter la contradiction quaternaire, l'objet de l'activité doit être négocié entre les systèmes. Cette négociation favorisera la création d'un objet potentiellement partagé entre les individus. La résolution des contradictions quaternaires permettra la consolidation du modèle et mènera à la transformation durable de l'activité collective (Engeström, 1987 ; Engeström, 2001 ; Viau-Guay \& Hamel, 2017).

\section{Figure 2}

Modélisation d'un possible cycle d'expansion illustrant la possibilité d'innovation des pratiques enseignantes dans un contexte problématique

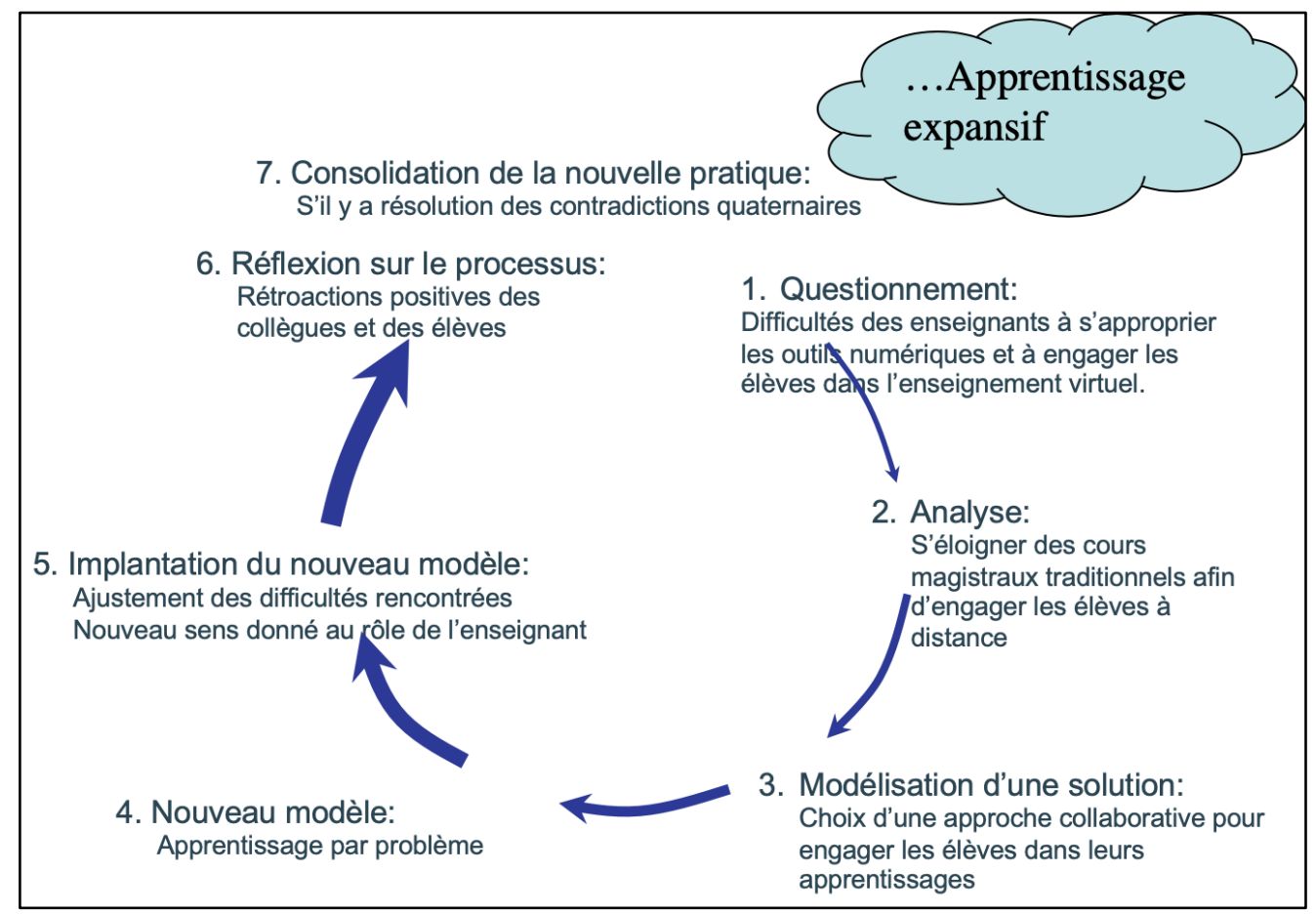


Revue internationale du CRIRES: innover dans la tradition de Vygotsky (2021) ISSN 2291-6717, vol 5, no 2, 63-73

Le contexte de la pandémie mondiale comme source possible d'innovation: apprentissage expansif et résolution de contradictions

La figure 2 résume, à l'aide du cycle d'expansion modélisé par Engeström (1987), les actions d'apprentissage possibles des enseignants qui désirent engager les élèves lors des périodes d'enseignement virtuel durant la pandémie de la COVID-19. Bien que l'illustration du cycle laisse croire que le processus est linéaire, lors de la résolution d'une contradiction, plusieurs allers-retours sont possibles entre les diverses actions d'apprentissage (questionnement, analyse, etc.) (Viau-Guay \& Hamel, 2017).

\section{Conclusion}

Selon un rapport des Nations Unies, «la pandémie de COVID-19 a provoqué la plus grande perturbation des systèmes éducatifs de l'histoire»(2020, p. 5). Cette situation bouleversante a forcé le milieu scolaire à réévaluer et à changer ses pratiques en peu de temps afin de s'adapter à la nouvelle réalité virtuelle. Bien que la demande fût forte sur les enseignants lors du passage au virtuel (Departement for education, 2020), certains en ont profité pour « réfléchir sur comment l'utiliser afin d'améliorer l'expérience éducative des élèves postCOVID-19» (Kim \& Asbury, 2020, p. 1062). L'analyse systématique des écrits montre que le contexte de la pandémie peut être favorable à l'apprentissage expansif des enseignants puisqu'il peut entrainer l'apparition de conflits inattendus chez ceux-ci, contribuer à la remise en question des pratiques enseignantes et ainsi favoriser l'innovation. Toutefois, les transformations expansives sont un long processus qui nécessite de résoudre plusieurs contradictions. L'apprentissage ne sera pas immédiat, il sera construit par la transformation qualitative du système d'activité entier (Daniels, 2004 ; Goodnough \& Murphy, 2017). Bien qu'éprouvantes, la résolution des conflits et la création de nouveaux savoirs permettront de transformer de manière profonde la pratique (Engeström \& Pyorala, 2021 ; Engeström \& Sannino, 2010).

Afin d'être mieux préparé aux prochains défis, il importe de continuer à innover les pratiques éducatives et à faire preuve de créativité et de souplesse dans les façons d'éduquer les jeunes (Nations Unies, 2020). Les enseignants conserveront-ils les apprentissages qu'ils ont faits durant la pandémie afin de favoriser l'apprentissage et la réussite éducative de leurs élèves ?

\section{Limites de l'étude}

Il importe de préciser quelques limites à l'étude. Tout d'abord, l'analyse concerne uniquement les pratiques enseignantes occidentales. En effet, la majorité des études recensées provient du Québec, de la France et du Royaume-Uni. De plus, la recension systématique des écrits n'a pris en compte que les études traitant des difficultés relatives à l'enseignement virtuel. Il serait intéressant d'explorer également les avantages de cette modalité d'enseignement afin de savoir si elles peuvent contribuer à l'innovation des pratiques enseignantes.

\section{Références}

Barma, S. (2008). Un contexte de renouvellement de pratiques en éducation aux sciences et aux technologies : Une étude de cas réalisée sous l'angle de la théorie de l'activité [Thèse de doctorat, Université Laval]. Corpus. https://corpus.ulaval.ca/jspui/handle/20.500.11794/20215

Barma, S. (2010). Analyse d'une démarche de transformation des pratiques en sciences, dans le cadre du nouveau programme de formation au secondaire, à la lumière de la théorie de l'activité. Canadian Journal of Education, 33(4), 677-710. 
Revue internationale du CRIRES: innover dans la tradition de Vygotsky (2021) ISSN 2291-6717, vol 5, no 2, 63-73

Le contexte de la pandémie mondiale comme source possible d'innovation: apprentissage expansif et résolution de contradictions

Berger, R. et Paul, M.S. (2021). Pedagogy vs. Technology: Challenges In Developing Online Courses in Social Work Education. Journal of Teaching in Social Work, 41(3), 275-289. https://doi.org/10.1080/08841233.2021.1919280

Besser, A., Flett. G. L., et Zeigler-Hill, V. (2020). Adaptability to a Sudden Transition to Online Learning During the COVID-19 Pandemic: Understanding the Challenges for Students. Scholarship of Teaching and Learning in Psychology. 10.1037/st10000198

Brassac, C. (2003). Lev, Ignace Jerome et les autres... Vers une perspective constructiviste en psychologie interactionniste. Technologies, Idéologies et Pratiques: revue d'anthropologie des connaissances, 15 (1), 195-214.

Daniels, H. (2004). Cultural historical activity theory and professional learning. International Journal of Disability, Development and Education, 51(2), 185-200.

Department for Education (2020). Adapting teaching practice for remote education. https://www.gov.uk/guidance/adapting-teaching-practice-for-remote-education

Deslandes, R., Barma, S., et Beaumier, F. (2020). Collaborative school-family relationships using the expansive learning cycle to enhance parents empowering in helping their child in the context of a new reading method. International Journal about parents in education, 12(1), 47-61.

Durak, G. et Çankaya, S. (2020). Undergraduate Students' Views About Emergency Distance Education During the Covid-19 Pandemic. European Journal of Open Education and E-learning Studies, 5(1), 122-147.

Engeström, Y. (1987). Learning by expanding: An activity-theoretical approach to developmental research. Orienta-Konsultit.

Engeström, Y. (1999). Innovative learning in work teams. Analyzing cycles of knowledge creation in practice, dans Y. Engeström, R. Miettinen et R. -L. Punamäki (dir.), Perspectives on activity theory (p. 377-404). Cambridge University Press.

Engeström, Y. (2001). Expansive learning at work: Toward an activity-theoretical reconceptualization. Journal of Education and Work, 14, 133-156.

Engeström, Y. et Pyorala, E. (2021). Using activity theory to transform medical work and learning. Medical Teacher, 43(1), 7-13. 10.1080/0142159X.2020.1795105

Engeström, Y. et Sannino, A. (2010). Studies of expansive learning: Foundations, findings and future challenges. Educational Research Review, 5(1), 1-24.

Gopalan, C., Butts-Wilmsmeyer, C., \& Moran, V. (2021). Virtual flipped teaching during the COVID-19 pandemic. Advances in Physiology Education, 45 (4), 670-678. https://doiorg.acces.bibl.ulaval.ca/10.1152/advan.00061.2021

Goodnough, K. et Murphy, E. (2017). An analysis of the professional learning of science teachers using the metaphor of learning by expanding. Educational Research, 27(1), 6481.

Gouvernement du Canada. (2019). Le gouvernement du Canada se mobilise contre la COVID19. https://www.canada.ca/fr/sante-publique/services/maladies/2019-nouveaucoronavirus/reponse-canada/gouvernement-canada-prend-mesures-concernant-covid19.html

Gouvernement du Canada. (2021). Fermeture d'écoles : les perturbations liées à la pandémie mettent en évidence les défis et les solutions pour les apprenants du monde entier. https://www.international.gc.ca/world-monde/stories-histoires/2021/education_covid19.aspx?lang=fra

Gouvernement du Québec. (2020). Le gouvernement du Québec déclare l'état d'urgence sanitaire, interdit les visites dans les centres hospitaliers et les CHSLD et prend des mesures spéciales pour offrir des services de santé à distance. https://www.quebec.ca/premier-ministre/actualites/detail/le-gouvernement-du-quebecdeclare-l-etat-d-urgence-sanitaire-interdit-les-visites-dans-les-centres-h/ 
Revue internationale du CRIRES: innover dans la tradition de Vygotsky (2021) ISSN 2291-6717, vol 5, no 2, 63-73

Le contexte de la pandémie mondiale comme source possible d'innovation: apprentissage expansif et résolution de contradictions

Kim, L. E., et Asbury, K. (2020). «Like a rug had been pulled from under you » : The impact of COVID-19 on teachers in England during the first six weeks of the UK lockdown. British Journal of Educational Psychology, 90(4), 1062-1083. 10.1111/bjep.12381

Koris, R., et Pál, Á. (2021). Fostering learners' involvement in the assessment process during the COVID-19 pandemic: Perspectives of university language and communication teachers across the globe. Journal of University Teaching \& Learning Practice, 18(5), $11-20$.

Leontiev, A. N. (1978). Activity, Consciousness, and Personality. Prentice Hall.

Martinez-Alvarez, P. (2019). What counts as science? Expansive learning actions for teaching and learning science with bilingual children. Cultural studies of science education, 14, 799-837.

Mullen, C., Pettigrew, J., Cronin, A., Rylands, L., et Shearman, D. (2021). The rapid move to online mathematics support: changes in pedagogy and social interaction. International Journal of Mathematical Education in Science \& Technology, 1-28. https://doiorg.acces.bibl.ulaval.ca/10.1080/0020739x.2021.1962555

Nations Unies. (2020). Note de synthèse: L'éducation en temps de COVID-19 et après. https://www.un.org/sites/un2.un.org/files/policy_brief__education_during_covid19 and beyond_french.pdf

Nicolet, M. (1995). Dynamiques relationnelles et processus cognitifs. Delachaux et Niestlé.

Özüdogru, G. (2021). Problems faced in distance education during Covid-19 pandemic. Participatory Educational Research, 8(4), 321-333. http://dx.doi.org/10.17275/per.21.92.8.4

Sannino, A. Engeström, Y., et Lemos, M. (2016). Formative Interventions for Expansive Learning and Transformative Agency. Journal of the Learning Sciences, 25(4), 599633. 10.1080/10508406.2016.1204547

Seke, M. M. (2020). Would we be able to absorb the new normal brought by COVID-19 as another educational revolution?. International Journal of Advanced Corporate Learning, 13(4), 68-87. 10.3991/ijac.v13i4.16525

Sève, L. (1985). Avant-Propos, dans L. S. Vygotski (dir.), Pensée et langage (p. 7-19). Messidor/Éditions Sociales.

Syauqi, K., Munadi, S., et Triyono, M. B. (2020). Students' Perceptions toward Vocational Education on Online Learning during the COVID-19 Pandemic. International Journal of Evaluation and Research in Education, 9(4), 881-886.

UNESCO (2020). Adverse consequences of school closures. https://en-unescoorg.acces.bibl.ulaval.ca/covid19/educationresponse/consequences

Viau-Guay, A., et Hamel, C. (2017). L'expérimentation de nouveaux modèles d'action pédagogique au sein d'une communauté professionnelle d'apprentissage en formation professionnelle. Revue Canadienne de l'Éducation, 40 (3), 123-157.

Vygotsky, L. S. (1985). Pensée et langage. Messidor/Éditions sociales.

Westwick, J. N., et Morreale, S. P. (2021). Advancing an agenda for instructional preparedness: lessons learned from the transition to remote learning. Communication Education, 70(2), 217-222. 10.1080/03634523.2020.1857416 